\title{
TERMOTERAPIA EM CÃES
}

Samyla Paiva de Albuquerque ${ }^{1}$; Ariel de Aguiar²; Lorena Oliveira da Silva ${ }^{2}$; Luís Eduardo Maggi ${ }^{3}$; Soraia Figueiredo de Souza ${ }^{4}$

1 Mestranda pelo Programa de Pós-Graduação em Sanidade e Produção Animal Sustentável na Amazônia Ocidental, Universidade Federal do Acre (UFAC). Rio Branco-AC, Brasil (samyla@hotmail.com)

2 Estudante de graduação de Medicina Veterinária, Universidade Federal do Acre 3 Professor Adjunto, Universidade Federal do Acre

4 Docente do Centro de Ciências Biológicas e da Natureza, Universidade Federal do Acre

Recebido em: 02/10/2017 - Aprovado em: 21/11/2017 - Publicado em: 05/12/2017 DOI: 10.18677/EnciBio 2017B62

A termoterapia é uma modalidade terapêutica que emprega diferentes métodos que utilizam o frio e/ou o calor para o tratamento de doenças ou traumas. Apesar de ser bastante difundida na Medicina Veterinária, é possível constatar que muitos protocolos de tratamento são empíricos ou extrapolados de resultados de pesquisas em humanos. Poucos estudos sobre as respostas às diferentes modalidades de termoterapia foram conduzidos em cães. A aplicação do frio ou crioterapia é indicada para reduzir os sinais da inflamação aguda. A utilização do calor é um dos procedimentos terapêuticos mais antigos, sendo uma terapia adjuvante na analgesia e no aumento de oxigenação tecidual local e da extensibilidade do tecido conjuntivo. Agentes de aquecimento superficial podem penetrar até $2 \mathrm{~cm}$ de profundidade, enquanto que o calor profundo até $5 \mathrm{~cm}$. O calor superficial pode ser aplicado através de compressas ou bolsas quentes, água morna e cobertores ou colchões aquecidos. Já o calor profundo vem sendo utilizado através da aplicação do ultrassom terapêutico. Um dos objetivos mais importantes de qualquer programa de reabilitação é alcançar uma completa amplitude de movimento. Técnicas de mobilização para tentar melhorar a condição muscular e a movimentação articular podem ser utilizadas, porém, está indicado o aquecimento prévio dos tecidos envolvidos. A termografia de infravermelho é um recurso de diagnóstico por imagem emergente indicada para aferir as variações térmicas de superfícies, podendo ser utilizada no monitoramento do aquecimento ou resfriamento superficial relacionados à utilização das modalidades de termoterapia.

PALAVRAS-CHAVE: crioterapia, calor, cão, ultrassom terapêutico 


\title{
THERMOTHERAPY IN DOGS
}

\begin{abstract}
Thermotherapy is a therapeutic modality employing different methods that use cold and / or heat for the treatment of diseases or traumas. Although it is widespread in veterinary medicine, it is possible to verify that many treatment protocols are empirical or extrapolated from human research results. Few studies on the responses to the different thermotherapy modalities were conducted in dogs. The application of cold or cryotherapy is indicated to reduce the signs of acute inflammation. The utilization of heat is one of the oldest therapeutic procedures, being an adjuvant therapy in analgesia and in the increase of local tissue oxygenation and the extensibility of connective tissue. Surface heating agents can penetrate up to $2 \mathrm{~cm}$ deep, while deep heat up to $5 \mathrm{~cm}$. The superficial heat can be applied through hot compresses or packs, warm water and heated blankets or mattresses. The deep heat has been used through the application of therapeutic ultrasound. One of the most important goals of any rehabilitation program is to achieve full range of motion. Mobilization techniques to try to improve muscular condition and joint movement can be used, however, the previous heating of the involved tissues is indicated. Infrared thermography is an emerging imaging diagnostic feature used to measure thermal variations of surfaces and can be used to monitor heating or surface cooling related to the use of thermotherapy modalities.
\end{abstract}

KEYWORDS: cryotherapy, dog, heat, therapeutic ultrasound

\section{INTRODUÇÃO}

Modalidades de agentes físicos têm sido utilizadas em reabilitação e fisioterapia por séculos para reduzir edema, aliviar a dor, melhorar a cicatrização, aumentar força e tônus muscular, e para modificar a elasticidade do tecido conjuntivo (HANKS et al., 2015). A termoterapia consiste na aplicação ou retirada do calor corporal para fins terapêuticos. Também pode ser definida como a utilização de calor e/ou frio superficiais e o calor profundo como modalidades terapêuticas para o tratamento de doenças ou traumas, e pode ser utilizada na fisioterapia veterinária empregando-se muitos métodos diferentes (MIKAIL; PEDRO, 2009; DRAGONE et al., 2014). O aumento ou a diminuição da temperatura em um tecido varia de acordo com o tipo de agente físico utilizado, o tempo de exposição, a natureza do tecido e a localização (LIAPI; GESCHWIND, 2007).

A aplicação do frio como método em reabilitação é denominada crioterapia e está relacionada à redução dos sinais cardinais da inflamação (dor, edema, hiperemia, aumento da temperatura e diminuição da função) (MIKAIL; PEDRO, 2009). Evidências clínicas e fisiológicas sugerem que a aplicação do frio em diversas formas induz vasoconstrição, diminui fluxo sanguíneo, espasmo muscular e edema tecidual, reduz o metabolismo e danos teciduais mediados por enzimas e promove analgesia pela diminuição da velocidade de condução nervosa (SAMOY et al., 2016).

Agentes de aquecimento superficial penetram até $2 \mathrm{~cm}$ de profundidade do tecido, enquanto que o calor profundo eleva a temperatura tecidual em profundidade de até $5 \mathrm{~cm}$ (DRAGONE et al., 2014). A terapia com o calor pode ser utilizada após o término da fase inflamatória aguda do processo de cicatrização, gerando dilatação dos vasos cutâneos, melhorando a extensibilidade dos tecidos conjuntivo e muscular, aumentando o limiar de dor e podendo ser útil antes da realização dos 
exercícios, alongamentos e para melhorar a amplitude de movimentos (STEISS; LEVINE, 2008).

Um dos objetivos mais importantes de qualquer tratamento ou programa de reabilitação é alcançar uma completa amplitude de movimento (AM). Diversos fatores limitantes como contraturas articulares, tecidos cicatriciais e aderências, podem tornar difícil atingir esse objetivo. Técnicas de mobilização para tentar melhorar a condição muscular e a movimentação articular podem ser utilizadas, porém está indicado o aquecimento prévio dos tecidos envolvidos (DRAPER; RICARD, 1995).

A termografia, um método emergente de aferição da temperatura da superfície do corpo com auxílio de câmera infravermelha, permite avaliar a resposta dessas diferentes modalidades. Dessa forma, as informações obtidas podem servir como base para a formulação de diferentes protocolos, uma vez que atualmente os mesmos muitas vezes são prescritos empiricamente ou com base em outras espécies de animais ou humanos. Com esta revisão de literatura objetivou-se descrever os principais métodos de termoterapia utilizados na medicina veterinária e suas indicações e contraindicações, e apontar o importante aspecto da tecnologia termográfica, que tem forte impacto no bem-estar animal pela sua natureza preventiva e não-invasiva.

\section{Termoterapia}

\section{REVISÃO DE LITERATURA}

O uso de alterações de temperatura teciduais no corpo, tanto frio como calor, talvez seja a forma mais antiga de tratamento que utiliza meios físicos com registros históricos da aplicação desses recursos para fins curativos (ARAÚJO, 2009). Comumente utilizadas como complemento ao exercício terapêutico, em adição a intervenções médicas e cirúrgicas, as modalidades de agentes físicos auxiliam a limitar deficiências e incapacidades e a maximizar funções. O mecanismo de ação e a profundidade de penetração variam de acordo com o método de aplicação e a forma de energia utilizada (condução, convecção ou conversão) (HANKS et al., 2015).

Embora haja um volume relativamente grande de literatura sobre as características e uso das modalidades de reabilitação, existem poucos estudos na espécie canina. Além disso, a maioria avalia características relacionadas com a mudança de temperatura do tecido causadas pelas modalidades de termoterapia, havendo ainda menos estudos avaliando a sua eficácia clínica (MILLIS; CIUPERCA, 2015).

Millard et al. (2013a) afirmam que a aplicação de calor para o tratamento de lesões dos tecidos moles ou dor crônica em cães é amplamente empírica, assim como a crioterapia, não havendo pesquisas baseadas em evidências neste assunto. Muller et al. (2009) comentam que, apesar do US terapêutico ser uma modalidade de tratamento comumente utilizada em fisioterapia de pequenos animais, há escassez de relatos científicos descrevendo o sucesso dessa técnica para lesões musculares de cães. Muitos protocolos para a administração do US são baseados em tradição ou extrapolados de pesquisa científica básica, sendo necessário ser testado em ensaios clínicos controlados (LEVINE; WATSON, 2014).

\section{- Crioterapia}

A crioterapia significa "terapia com frio", isto é, aplicação terapêutica de qualquer agente que remova o calor corporal, diminuindo a temperatura dos tecidos. 
Sendo assim, todas as técnicas que utilizam o frio como massagem com gelo, criocirurgia, crioalongamento, submissão de uma queimadura à agua fria são consideradas crioterapia (CORTI, 2014; KIEVES et al., 2016; RAMOS et al., 2016). Segundo Dragone et al. (2014), a aplicação de frio pode ser realizada de diversas maneiras: cobertores de água fria circulante, bolsas de gelo reutilizáveis, cubos de gelo enrolados em toalha, copos de gelo, imersão fria, compressas frias, sprays de vapor frio ou banhos de contraste.

A crioterapia é utilizada não somente durante uma fase aguda de lesão tecidual ou como tratamento para atenuar os efeitos e as sequelas da lesão, mas também após o exercício, durante a reabilitação para minimizar as respostas adversas secundárias à inflamação (DRAGONE et al., 2014). A diminuição da dor e da inflamação pode levar ao aumento da AM de articulações comprometidas. Além disso, a aplicação local do frio pode reduzir a espasticidade de desordens da medula espinhal (HANKS et al., 2015).

Drygas et al. (2011) afirmam que o objetivo terapêutico primário dessa modalidade é suprimir a taxa metabólica do tecido traumatizado ao diminuir a temperatura tecidual. Adicionalmente, a hipotermia diminui a dor pós-operatória por reduzir as concentrações do fator de necrose tumoral (TNF) e óxido nítrico (NO), que são dois mediadores inflamatórios importantes. Em seu estudo, observaram os efeitos da crioterapia após osteotomia de nivelamento do platô tibial: grau de dor significativamente menor, índice de claudicação mais baixo, menos edema e melhor AM nas primeiras 24 horas após a cirurgia.

O frio é frequentemente usado para o tratamento da dor, inflamação, hemorragia, inchaço e edema associado ao trauma de tecidos moles. O frio também diminui a circulação sanguínea por aumentar a viscosidade e a vasoconstrição, assim como diminui a velocidade de condução nervosa. A diminuição do metabolismo tecidual ajuda a proteger o tecido e a área circundante das reações enzimáticas associadas à lesão e inflamação subsequente, assim como a reduzir a liberação de histamina (MILLARD et al., 2013b; HANKS et al., 2015).

Millard et al. (2013b) afirmam que, apesar de não haver na literatura científica a definição da frequência ótima e duração do tratamento, o consenso geral é de que repetidas aplicações de compressa fria de 10 a 30 minutos são efetivas em melhorar os resultados clínicos em humanos. Geralmente, a aplicação de crioterapia em cães é empírica e tem sido extrapolada das recomendações para humanos. Em seu trabalho, ao estudar o efeito do uso de compressas frias $\left(-16^{\circ} \mathrm{C}\right)$ no músculo epaxial de cães, observaram que as diferenças entre as aplicações de 10 e de 20 minutos foram significativas, onde o tratamento de 20 minutos foi melhor, porém, as aplicações de 10 minutos foram suficientes para obter resfriamento com efeito terapêutico.

Dragone et al. (2014) afirmam que o tempo de tratamento com crioterapia depende da modalidade escolhida e da área a ser tratada, mas geralmente é de 15 a 20 minutos e pode ser repetida durante o dia. O método mais comum é repetir as aplicações do frio a cada duas a quatro horas durante as primeiras 24 a 48 horas após o trauma. O tratamento com gelo não deve ser aplicado por mais de 20 minutos por sessão para evitar danos ao tecido. A hipotermia local nunca deverá exceder os 30 minutos por sessão pelos mesmos motivos, como também para evitar a estimulação de vasodilatação local ou formação de edema não desejáveis. Millis e Ciuperca (2015) também afirmam que mudanças na temperatura tecidual e os efeitos adversos da aplicação por mais de 20 minutos requerem maiores investigações. 
Complicações após a crioterapia são incomuns e, se forem usados os cuidados necessários, elas são facilmente evitadas. As complicações mais frequentes são queimadura e paralisia nervosa. Áreas que já foram previamente acometidas devem ser evitadas. A crioterapia deve ser aplicada com cuidado sobre nervos superficiais, feridas abertas, fraturas, áreas onde podem haver danos aos nervos motores ou sensoriais, em pacientes com hipertensão, em áreas com sensibilidade diminuída ou ausente e em pacientes muito jovens ou muito velhos. $A$ crioterapia nunca deve ser realizada antes de atividade física vigorosa, pois o frio aumenta a rigidez das fibras colágenas, resultando em diminuição da flexibilidade muscular (DRAGONE et al., 2014; HANKS et al., 2015; CANAPP, et al., 2016).

\section{- Calor Superficial}

Poucos procedimentos terapêuticos têm sido historicamente utilizados tanto quanto o calor (DRAGONE et al., 2014). Essa terapia é uma ferramenta adjuvante no controle da dor, útil no tratamento das desordens musculoesqueléticas dos cães. $\mathrm{O}$ aquecimento dos tecidos promove mobilidade às unidades musculotendíneas, e há base teórica adicional para o uso do calor em melhorar a cicatrização de feridas crônicas. Os benefícios terapêuticos do calor superficial resultam dos seus efeitos em aumentar a oxigenação tecidual local e seu metabolismo, reduzindo espasmo muscular, aumentando extensibilidade do tecido conjuntivo e elevando o limar da dor (DORN, 2015a).

No calor superficial, as principais indicações são: analgesia, relaxamento muscular e aumento da amplitude articular (MIKAIL; PEDRO, 2009), podendo preceder exercícios e técnicas de manipulação (FURLAN et al., 2015). Os efeitos do calor na fisioterapia incluem hiperemia e dilatação dos vasos cutâneos, aumento na transferência de metabólitos pelas membranas dos capilares, relaxamento muscular geral, efeito sedativo e analgésico e melhora na extensibilidade do tecido conjuntivo (BISTNER ; KIRK , 2002). Quando aplicado antes de alongamento e exercício, pode haver menor dano tecidual e maior AM (MILLIS; CIUPERCA, 2015).

A temperatura tecidual deve ser elevada pelo menos 3 a $4^{\circ} \mathrm{C}$ acima do normal para que se obtenha aumento ótimo na extensibilidade do tecido associado à melhora da flexibilidade (LEVINE et al., 2001). Compressas quentes podem aquecer tecidos a uma profundidade de até $2 \mathrm{~cm}$. Contudo, a maior mudança de temperatura é observada somente desde a superfície da pele até aproximadamente $1 \mathrm{~cm}$ de profundidade (DYCUS et al., 2017).

Similarmente à crioterapia, o calor superficial é largamente utilizado com bastante variabilidade dos métodos, frequência e duração da aplicação. Segundo Millard et al. (2013a), a aplicação de compressas mornas deve ser realizada por 10 minutos. Em seu trabalho, não foram observados resultados significativos no aumento do aquecimento de nenhuma das profundidades avaliadas dos tecidos quando a duração da aplicação aumentou para 20 minutos. Já Dragone et al. (2014) indicam uma duração do tratamento com calor de 15 a 30 minutos, podendo ser repetido de três a quatro vezes ao dia. Dorn (2015b) afirma que ainda não foi confirmada a duração ótima para a aplicação de calor em cães e gatos, mas é provável que seja pelo menos 10 minutos.

Tratamentos com calor superficial incluem bolsas quentes, compressas quentes, cobertores com água quente circulante, colchões aquecidos e o uso de água morna por imersão total ou parcial do corpo (DRAGONE et al., 2014). A aplicação terapêutica do calor não deve causar dor. A temperatura máxima sugerida a ser aplicada sobre a pele de cães e gatos é de $41^{\circ} \mathrm{C}$, apesar de essa temperatura 
poder ser nociva a alguns pacientes. Portanto, o monitoramento de perto do comportamento do animal é essencial, retirando ou ajustando a fonte de calor caso seja necessário (DORN, 2015b). Já Millis e Ciuperca (2015) relatam que a temperatura ótima de compressa para alcançar níveis terapêuticos benéficos ainda é desconhecida. $O$ paciente deve ser protegido de queimaduras isolando-se as bolsas térmicas com toalhas e examinando-se a pele constantemente. A terapia com o calor superficial deve ser modificada ou suspensa se causar dor ou houver exacerbação dos sinais patológicos. São necessárias precauções para pacientes gestantes, extremamente obesos ou magros, circulação prejudicada, regulação térmica ineficiente, insuficiência cardíaca e pacientes muito jovens ou muito velhos (DRAGONE et al., 2014; CANAPP et al., 2016).

As principais contraindicações do uso do calor são: fase aguda de processos inflamatórios e pacientes termo e fotossensíveis (MIKAIL; PEDRO, 2009). A aplicação local do calor também é contraindicada nas situações de sangramento agudo, tromboflebite, febre e presença de inchaço ou edema (DRAGONE et al., 2014). A tolerância do paciente ao calor e ao frio pode estar significativamente alterada em casos de dor crônica, especialmente em áreas de alodinia. Para pacientes que estão incapacitados de mudar de posição, vocalizar ou demonstrar outros sinais de desconforto e para aqueles com a sensação dérmica comprometida, o aquecimento superficial terapêutico não pode ser seguramente monitorado e, por esse motivo, é contraindicado (DORN, 2015b).

\section{- Calor profundo}

O tratamento com calor profundo, como o ultrassom térmico e a diatermia, penetra no corpo para alcançar tecidos mais distantes (DORN, 2015a), podendo elevar a temperatura dos tecidos à profundidade de $2 \mathrm{~cm}$ ou mais. $\mathrm{O}$ calor profundo vem sendo utilizado na fisioterapia veterinária através do ultrassom (US) terapêutico, sendo este considerado uma modalidade de tratamento efetivo para reabilitação de condições musculoesqueléticas como AM restrita (LEVINE; WATSON, 2014; SIMS et al., 2015).

Os efeitos terapêuticos dessa modalidade podem derivar primariamente do aquecimento do tecido e do aumento da elasticidade do colágeno (SIMS et al., 2015). As principais indicações do US terapêutico são tecidos moles encurtados (contratura, cicatriz), inflamação subaguda ou crônica e dor (pontos-gatilho) (LEVINE; WATSON, 2014).

Casos envolvendo imobilização articular ou repouso por períodos prolongados são caracterizados pela diminuição da elasticidade muscular e redução da AM. Essa condição, conhecida como contratura, é possivelmente a complicação mais frequentemente encontrada pelos profissionais de reabilitação. Geralmente, alongamento prolongado e/ou termoterapia são aplicados em programas de reabilitação de contratura muscular com o objetivo de melhorar a extensibilidade do músculo esquelético (KONDO et al., 2012; SIMS et al., 2015).

A AM normal de uma articulação é mantida por repetidos movimentos de sua respectiva parte do corpo diariamente. Forças físicas são necessárias para estimular a síntese de novas fibras de colágeno. A imobilização ou qualquer incidente que resulte no desuso da articulação afetada irá privá-los da força física oposta que mantém a AM normal, resultando em contratura articular. O calor é utilizado também no tratamento de contraturas articulares, quando o encurtamento da cápsula articular e das estruturas periarticulares ocorrem, pois o calor aumenta efetivamente a extensibilidade das fibras de colágeno do tecido (USUBA et al., 2006). 
Uma das modalidades que usualmente é aplicada com o objetivo de diminuir aderências é o US, indicado nas condições em que a cicatrização resulta em restrição da AM. É possível aumentar a extensibilidade do tecido conjuntivo quando a sua temperatura é elevada entre $39^{\circ} \mathrm{C}$ a $47^{\circ} \mathrm{C}$, onde quanto maior o aquecimento, maior a extensão resultante. Quando o objetivo é aumentar as propriedades viscoelásticas do colágeno para que o tecido seja alongado, é necessário aquecimento vigoroso, maior que $3^{\circ} \mathrm{C}$ (DRAPER; RICARD, 1995).

Usuba et al. (2006) concluíram em seu estudo que, para a recuperação mais rápida da $\mathrm{AM}$ após o desenvolvimento de contratura articular, o uso do calor é recomendado e a escolha da modalidade de termoterapia pode ser tanto superficial como profunda. Em contrapartida, Kondo et al. (2012) afirmam que as evidências que suportam o uso de alongamento prolongado e termoterapia para contratura muscular são incompletas, não tendo observado em seu trabalho efeitos significativos da termoterapia no processo de recuperação da contratura muscular.

O US terapêutico pode ser aplicado de forma contínua ou pulsátil, dependendo do tipo de enfermidade em tratamento. A forma contínua produz 50\% de efeito térmico e $50 \%$ de efeito mecânico, e o US pulsado produz ação mecânica sem produzir calor (atérmico) (MACHADO, 2002), apesar de Itakura et al. (2012) afirmar que não é possível separar ambos efeitos. O efeito térmico pode reduzir a dor e o espasmo muscular, e elevar a capacidade de extensibilidade do colágeno (HENDERSON et al., 2015). O aquecimento produzido pela propagação do US nos tecidos vivos é um fenômeno complexo. A produção de calor resulta da interação entre as propriedades acústicas e térmicas dos tecidos e a onda irradiada (MAGGI et al., 2008). As ondas provenientes do US são absorvidas pelos tecidos e transformadas em calor, ocorrendo principalmente em nível molecular, sendo as proteínas dos tecidos que mais absorvem. No tecido ósseo, aproximadamente $70 \%$ das ondas incidentes são absorvidas (MACHADO, 2002).

O modo contínuo promove o aumento da temperatura dos tecidos com consequente aumento da extensibilidade do colágeno, do fluxo sanguíneo local, da velocidade de condução do estímulo nervoso e do limiar de dor, reduzindo espasmo, contratura muscular e desfazendo aderências (STEISS; LEVINE, 2008). Essa modalidade pode ser benéfica a pacientes com osteoartrite associada a displasia coxofemoral por melhorar a AM e diminuir a dor e o espasmo muscular, pois permite o aquecimento de tecidos profundos que não são alcançados pelo aquecimento por compressas quentes (DYCUS et al., 2017).

A elevação de temperatura gerada pelo US terapêutico depende de múltiplos fatores, incluindo intensidade de distribuição, tempo de exposição e as propriedades acústicas e térmicas do tecido tratado (DEMMINK et al., 2003; MILLIS; CIUPERCA, 2015). Devido ao fato de os tecidos dos mamíferos variarem em estrutura, as propriedades térmicas e acústicas dos tecidos diferem entre as espécies. Portanto, é bem provável que o grau de variação de temperatura atingido do tecido, utilizandose um determinado protocolo de US terapêutico, varie entre as espécies (MONTGOMERY et al., 2013). Deve-se levar em consideração o tecido a ser tratado, se tem maior ou menor quantidade de tecido adiposo cobrindo o músculo ou áreas com proeminências ósseas. $O$ tecido adiposo absorve menos ultrassom do que a derme e os músculos e tecidos com alto conteúdo proteico se aquecem muito mais que tecidos adiposos ou epiteliais (LEVINE et al., 2001; ITAKURA et al., 2012).

Uma variedade de frequências do US tem sido estudada nos humanos, sendo as mais comuns $1,0 \mathrm{MHz}$ e $3,3 \mathrm{MHz}$. A primeira tem os seus maiores efeitos na profundidade tecidual de 2,5 a $5 \mathrm{~cm}$. Na frequência de $3,3 \mathrm{MHz}$, os maiores efeitos 
ocorrem de 1 a 2,5 cm de profundidade (LEVINE et al., 2001). Segundo Levine e Watson (2014), é a frequência, e não a intensidade, que determina a profundidade de penetração. À medida que a frequência aumenta, a profundidade diminui. Porém, Maggi et al. (2008) afirmam ser importante ressaltar que, apesar de na frequência inferior $(1 \mathrm{MHz})$ o aumento de temperatura atingir camadas mais profundas, a elevação de temperatura nos centímetros iniciais dos tecidos é mais proeminente com a utilização de frequência superior $(3 \mathrm{MHz})$, ou seja, além do aquecimento ser mais superficial, também é mais intenso, podendo ultrapassar $46^{\circ} \mathrm{C}$ e causar desnaturação proteica.

O valor do aumento da temperatura tecidual está diretamente relacionado com a intensidade do US (LEVINE et al., 2001). Quanto mais alta a intensidade, maior e mais rápida a elevação da temperatura. A intensidade é referente à quantidade de energia liberada por unidade de área e pode variar de 0,25 a 3,0 $\mathrm{W} / \mathrm{cm}^{2}$ (LEVINE; WATSON, 2014). Levine et al. (2001), ao avaliar os efeitos do aquecimento do US terapêutico de 3,3 $\mathrm{MHz}$ na coxa de cães, escolheram utilizar intensidades de 1 e $1,5 \mathrm{~W} / \mathrm{cm}^{2}$ devido já ter sido previamente demonstrado que estas elevam as temperaturas teciduais sem causar desconforto em humanos. Os resultados encontrados foram que, utilizando um tratamento de 10 minutos, na intensidade de $1 \mathrm{~W} / \mathrm{cm}^{2}$ a temperatura tecidual aumentou $2-4^{\circ} \mathrm{C}$ nas profundidades de 1 e $2 \mathrm{~cm}$. Na intensidade de $1,5 \mathrm{~W} / \mathrm{cm}^{2}$, o aumento foi de pelo menos $2-4^{\circ} \mathrm{C}$ nas profundidades de 1,2 e $3 \mathrm{~cm}$.

Itakura et al. (2012) comentam que é necessário que o US terapêutico produza aquecimento no tecido alvo entre 40 e $45^{\circ} \mathrm{C}$, por aproximadamente 5 minutos, para que o efeito térmico desejado seja obtido. Podem ocorrer danos aos tecidos nas temperaturas acima de $45^{\circ} \mathrm{C}$. Segundo Maggi et al. (2008), efeitos terapêuticos podem não ser alcançados a temperaturas inferiores a $40^{\circ} \mathrm{C}$.

Dentre as vantagens do US terapêutico está a produção de calor local em tecidos mais profundos e o tempo mais curto de tratamento, de aproximadamente 10 minutos. Como desvantagens, a dosagem é de difícil monitoramento e o contato do transdutor com a pele pode agravar a sensibilidade preexistente da área tratada (LEVINE; WATSON, 2014). A intensidade de saída, as especificidades do tratamento e a dimensão da área a ser tratada determinam a duração do tratamento (ITAKURA et al., 2012). A terapia pode ser administrada uma a duas vezes ao dia em pacientes com dor intensa ou espasmos. Pacientes que não estão severamente afetados requerem menos sessões (SIMS et al., 2015).

O US é contraindicado nos seguintes locais e/ou afecções: marcapassos cardíacos, olhos, baço, cérebro, coração, dermatomiosite, diabetes, epífise óssea em crescimento, fígado, infecção renal e urinária, órgãos reprodutores, útero gravídico, processo inflamatório agudo, áreas lesionadas imediatamente após o exercício, feridas contaminadas, locais de incisão recente, sobre a coluna e tumores (LEVINE; WATSON, 2014; CANAPP, et al., 2016).

\section{Termografia}

No campo da medicina veterinária, muitas técnicas têm surgido com o intuito de facilitar cada vez mais os diferentes procedimentos, trazendo benefícios à clínica médica e também agregando mais valor científico às pesquisas. Com a importância que o bem-estar animal tem assumido nessa área, tornou-se imprescindível a utilização de técnicas e equipamentos não invasivos que prezem pelo conforto e bem-estar animal, destacando-se assim, a termografia de infravermelho (ROBERTO; SOUZA, 2014). Essa modalidade diagnóstica vem ganhando espaço como uma 
forma de técnica não-invasiva de mensuração térmica, com a vantagem de não mudar a temperatura superficial por não requerer contato direto com o paciente, além de ser possível a leitura instantânea da temperatura real da superfície corpórea (BIONDI et al., 2015).

Esse método fornece informações sobre a saúde do animal detectando condições inflamatórias e infecções, assim como sobre o estresse agudo e crônico em animais de laboratório, de produção e esportivos, por haver uma estreita relação entre o estresse e o sistema metabólico (TRAVAIN et al., 2015).

A termografia é uma técnica de mapeamento térmico na qual a energia emanada pela superfície de um material ou corpo passa a ser o procedimento diagnóstico através da formação de imagem infravermelha. A emissão de radiação térmica natural ocorre devido à movimentação interna das moléculas de qualquer corpo ou objeto que apresentem temperatura acima de zero absoluto (DEY et al., 2017). Essa emissividade sofre influência de fatores como o ar, a umidade e a circulação sanguínea, e aumenta à medida que a circulação sanguínea periférica também aumenta (DE LIMA et al., 2013).

As radiações são capturadas por câmeras infravermelhas e transformadas em um termograma (mapa térmico) (PANDELEA; BUDESCU, 2016). As imagens representam áreas onde possam haver variações anormais de temperatura, demonstradas através de uma escala colorimétrica, na qual diferentes cores correspondem a uma temperatura específica e não à verdadeira cor do objeto (REDAELLI et al., 2014).

As cores encontradas nas imagens da termografia são: violeta, azul, verde, laranja, vermelho, amarelo e branco. Cores escuras, como roxo, azul e verde são específicas a baixas temperaturas, surgindo como pontos frios (cold spots), podendo estar associados a áreas com baixa perfusão tecidual, como em regiões de necrose e fibrose. O restante das cores claras correspondem às altas temperaturas, evidenciando a hipertermia como pontos quentes (hot spots), podendo estar clinicamente relacionados a áreas de inflamação, com aumento da circulação local e da taxa metabólica (PANDELEA; BUDESCU, 2016).

Em humanos, esse recurso é utilizado em diversas especialidades médicas como a angiologia, reumatologia, medicina esportiva, cirurgia plástica e na oncologia, especialmente para diagnóstico de câncer de mama em mulheres (PAVELSKI et al., 2015). A aplicação deste recurso é relativamente recente na veterinária. A principal vantagem de se utilizar métodos não invasivos é evitar qualquer interferência entre a observação e o comportamento espontâneo do animal. É considerada uma ferramenta de imagem não-invasiva por não causar danos ao paciente ou ao operador (REDAELLI et al., 2014). A termografia deve ser considerada um método de exame complementar utilizado para auxiliar o diagnóstico clínico, não devendo ser indicada para a realização de diagnóstico definitivo (LAHIRI et al., 2012).

Travain et al. (2016) utilizaram a termografia de infravermelho em combinação com medidas comportamentais, como frequência cardíaca e variabilidade da frequência cardíaca, para investigar as respostas emocionais dos cães ao receber alimento dos seus donos. Foram observados aumentos de temperatura ocular durante a estimulação positiva, apontando para a utilidade da termografia como ferramenta na avaliação do estado emocional de cães.

Kim e Park (2012) relataram um caso que foi possível detectar, através do exame termográfico, a localização de um trombo arterial no membro pélvico direito de um cão. O paciente apresentou paralisia aguda do membro e o termograma 
indicou redução considerável da temperatura na região abaixo da origem da artéria femoral profunda direita comparada ao membro pélvico esquerda. A suspeita diagnóstica foi confirmada pela incisão local da artéria, sendo então realizado o tratamento.

Biondi et al. (2015) avaliaram através da termografia de infravermelho a superfície córnea de cães normais e de cães com ceratoconjuntivite seca (CKS) com o objetivo de estabelecer os valores térmicos de referência e de explorar possíveis alterações na temperatura da superfície ocular em situações onde há um estado de baixa produção lacrimal. Observou-se que a termografia é um método que pode diferenciar ambas condições de normalidade e de deficiência de produção lacrimal, podendo futuramente ser incorporada como parte do exame oftálmico de pequenos animais e até se tornar um teste auxiliar popular para o diagnóstico de desordens da superfície ocular.

A termografia também tem sido indicada para mensuração e monitoramento da variabilidade térmica gerada pelo US (Figura 1). Como observado no estudo de Itakura et al. (2012), a obtenção do aquecimento profundo exige o controle de diversas variáveis. O resultado terapêutico pode ser influenciado caso a temperatura não atinja o valor ideal.

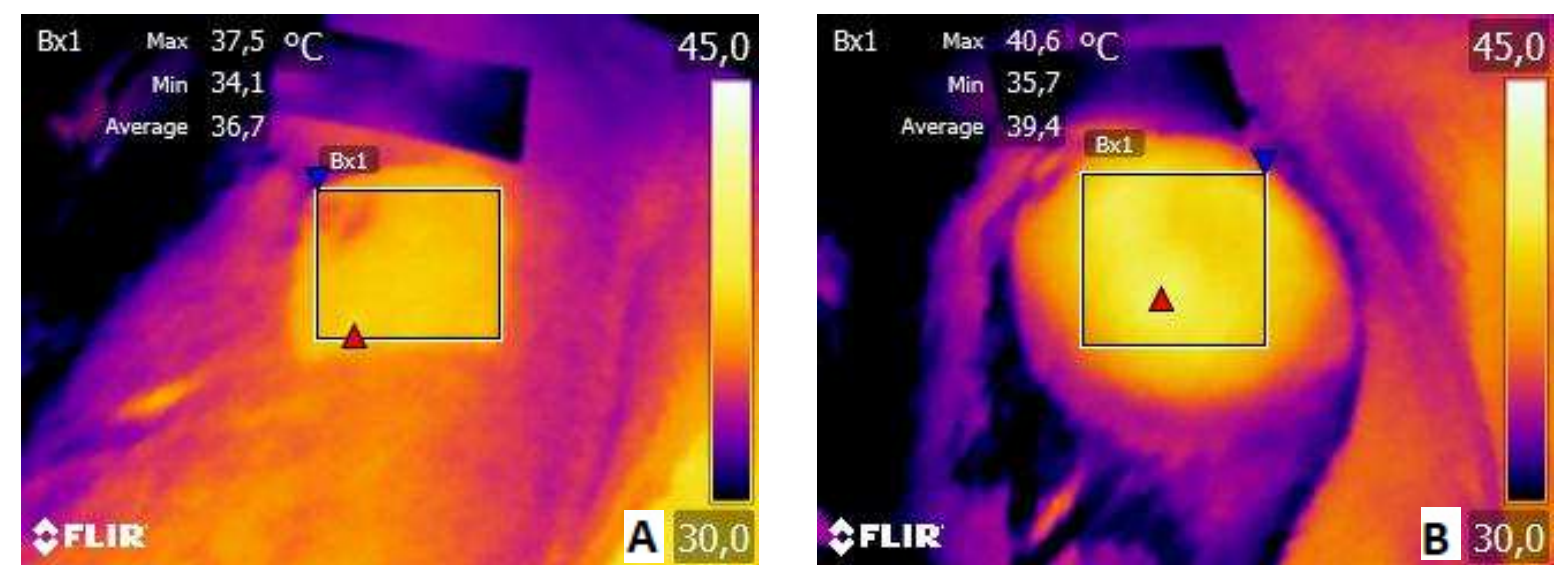

FIGURA 1 - Termografia do membro pélvico direito de cão. A - imediatamente antes da aplicação do ultrassom terapêutico. B - imediatamente após a aplicação do ultrassom terapêutico.

Fonte: Arquivo pessoal.

A identificação com precisão do local afetado é prejudicada pela falta de especificidade da termografia, pois a difusão do calor é dificultada através dos tecidos adiposo e ósseo, não sendo possível obter detalhes anatômicos sobre estruturas e órgãos mais internos (REDAELLI et al., 2014). Os resultados podem ser comprometidos pelo tipo de tecido avaliado. $O$ tecido adiposo é um isolante térmico efetivo, gerando leituras de baixas temperaturas. Assim, a termografia de indivíduos obesos pode ser difícil ou até impossível. O método mede apenas o calor emitido pela pele, o que significa que condições patológicas localizadas em tecidos mais profundos ou condições próximas à superfície da pele, que emitem apenas limitada quantidade de calor, não serão detectados (CHRISTENSEN et al., 2012).

Além disso, a imagem térmica de um animal muitas vezes apresenta anomalias térmicas não diretamente relacionadas ao seu estado fisiopatológico, mas devido a outras causas, tais como condições ambientais ou de medida, ou a variabilidade individual. Todavia, menciona-se que seu uso está sendo bastante 
recomendado por apresentar baixo custo, não ter necessidade de proceder sedação e por não oferecer risco de radiação (REDAELLI et al., 2014).

\section{CONSIDERAÇÕES FINAIS}

Através da revisão de literatura foi possível observar pequena quantidade de publicações que avaliam os efeitos dos diferentes métodos de aplicação de calor e/ou frio em cães, evidenciando uma lacuna que precisa ser preenchida na literatura neste campo de conhecimento. Pode-se concluir que as modalidades de termoterapia superficial têm seus protocolos de administração extrapolados de resultados encontrados em pesquisas em humanos, apesar de serem largamente utilizadas na Veterinária. É necessário realizar mais estudos sobre os efeitos dessas diferentes modalidades nos cães e assim poder elaborar protocolos específicos para a espécie. A termografia é uma tecnologia emergente que pode ser usada para avaliação e monitoramento das variações térmicas corporais superficiais de forma não-invasiva, sem causar desconforto ou estresse nos pacientes. Contudo, deve-se levar em consideração as variações normais de temperatura da pele e os diferentes escores corporais entre os pacientes.

\section{REFERÊNCIAS}

ARAÚJO, M. A. Termoterapia. In: Mikail, S.; Pedro, C. R., editors. Fisioterapia Veterinária. 2. ed. São Paulo: Manole, p. 76-88, 2009.

BIONDI, F.; DORNBUSCH, P. T.; SAMPAIO, M.; MONTIANI-FERREIRA, F. Infrared ocular thermography in dogs with and without keratoconjunctivitis sicca. Veterinary Ophthalmology, v. 18, n. 1, p. 28-34, 2015. Disponível em: <http://doi.org/10.1111/vop.12086>. doi: 10.1111/vop.12086

BISTNER, S. I.; KIRK, R. W. Manual de procedimentos veterinários e tratamento de emergência. 7. ed. São Paulo: Roca, p. 934, 2002.

CANAPP, D.; FAIR, L. M.; CANAPP, S. Complications Associated with Rehabilitation Modalities. In: Griffon, D.; Hamaide, A., editors. Complications in Small Animal Surgery. 1st edition. Wiley-Blackwell, p. 118-125, 2016.

CHRISTENSEN, J.; MATZEN, L. H.; VAETH, M.; SCHOU, S.; WENZEL, A. Thermography as a quantitative imaging method for assessing postoperative inflammation. Dentomaxillofacial Radiology, v. 41, n. 6, p. 494-499, 2012. doi: $10.1259 / \mathrm{dmfr} / 98447974$

CORTI, L. Nonpharmaceutical Approaches to Pain Management. Topics in Companion Animal Medicine, v. 29, n. 1, p. 24-28, 2014. Disponível em: <https://doi.org/10.1053/j.tcam.2014.04.001>. doi: 10.1053/j.tcam.2014.04.001

DE LIMA, V.; PILES, M.; RAFEL, O.; LÓPEZ-BÉJAR, M.; RAMÓN, J., et al. Use of infrared thermography to assess the influence of high envir/onmental temperature on rabbits. Research in Veterinary Science, v. 95, n. 2, p. 802-810, 2013. Disponível em: <http://doi.org/10.1259/dmfr/98447974 >. doi: 10.1016/j.rvsc.2013.04.012.

DEMMINK, J. H.; HELDERS, J. M. P.; HOBAEK, H.; ENWEMEKA, C. The variation of heating depth with therapeutic ultrasound frequency in physiotherapy. Ultrasound 
in Medicine \& Biology, v. 29, n. 1, p. 113-118, 2003. Disponível em: <https://doi.org/10.1016/S0301-5629(02)00691-9>. doi: 10.1016/S03015629(02)00691-9

DEY, N.; ASHOUR, A. S.; ALTHOUPETY, A. S. Thermal Imaging in Medical Science. In: Santhi, $V$, editor. Recent advances in applied thermal imaging for industrial applications. Pensilvania: IGI Global, p. 87-117, 2017.

DORN, M. Superficial heat therapy for dogs and cats, part 1: physiological mechanisms and indications. Companion Animal, v. 20, n. 12, p. 630-635, $2015 \mathrm{a}$. Disponível em: <https://doi.org/10.12968/coan.2015.20.11.630>. doi: 10.12968/coan.2015.20.11.630

DORN, M. Superficial heat therapy for dogs and cats, part 2: using heat therapy in practice. Companion Animal, v. 20, n. 12, p. 676-684, 2015b. Disponível em: $<$ https://doi.org/10.12968/coan.2015.20.12.676>. doi: 10.12968/coan.2015.20.12.676

DRAGONE, L.; HEINRICHS, K.; LEVINE, D.; TUCKER, T.; MILLIS, D. Superficial thermal modalities. In: Millis, D.; Levine, D., editors. Canine rehabilitation and physical therapy. 2nd edition. Philadelphia: Saunders, p. 312-327, 2014.

DRAPER, D. O.; RICARD, M. D. Rate of Temperature Decay in Human Muscle Following $3 \mathrm{MHz}$ Ultrasound: The Stretching Window Revealed. Journal of Athletic Training, v. 30, n. 4, p. 304-307, 1995. Disponível em: <https://www.ncbi.nlm.nih.gov/pmc/articles/PMC1317998/?page=1>.

DRYGAS, K. A.; MCCLURE, S. R.; GORING, R. L.; POZZI, A.; ROBERTSON, S. A., et al. Effect of cold compression therapy on postoperative pain, swelling, range of motion and lameness after tibial plateau leveling osteotomy in dogs. Journal of American Veterinary Medical Association, v. 238, n. 10, p. 1284-1291, 2011. Disponível em: <https://doi.org/10.2460/javma.238.10.1284>. doi: 10.2460/javma.238.10.1284.

DYCUS, D. L.; LEVINE, D.; MARCELLIN-LITTLE, D. J. Physical Rehabilitation for the Management of Canine Hip Dysplasia. Veterinary Clinics of North America: Small Animal Practice, v. 47, n. 4, p. 823-850, 2017. Disponível em: <https://doi.org/10.1016/j.cvsm.2017.02.006>. doi: 10.1016/j.cvsm.2017.02.006

FURLAN, R. M. M. M.; GIOVANARDI, R. S.; BRITTO, A. T. B. O.; BRITTO, D. B. O. $O$ emprego do calor superficial para tratamento das disfunções temporomandibulares: uma revisão integrativa. CoDAS, São Paulo, v. 27, n. 2, p. 207-212, 2015. Disponível em: <http://dx.doi.org/10.1590/2317-1782/20152014148>. doi: 10.1590/2317-1782/20152014148.

HANKS, J.; LEVINE, D.; BOCKSTAHLER, B. Physical agent modalities in physical therapy and rehabilitation of small animals. Veterinary Clinics of North America: Small Animal Practice, v. 45, n. 1, p. 29-44, 2015. Disponível em: <https://doi.org/10.1016/j.cvsm.2014.09.002>. doi: 10.1016/j.cvsm.2014.09.002 
HENDERSON, A. L.; LATIMER, C.; MILLIS, D. L. Rehabilitation and physical terapy for selecte orthopedic conditions in veterinary patients. Veterinary Clinics of North America: Small Animal Practice, v. 45, n. 1, p. 91-121, jan 2015. Disponível em: <https://doi.org/10.1016/j.cvsm.2014.09.006>. doi: 10.1016/j.cvsm.2014.09.006

ITAKURA, D. A.; MAGAS, V.; NEVES, E. B.; NOHAMA, P. Alteração da temperatura nos tecidos biológicos com a aplicação do ultrassom terapêutico: uma revisão. Fisioterapia em Movimento, v. 25, n. 4, p. 857-868, 2012. Disponível em: <http://dx.doi.org/10.1590/S0103-51502012000400019>. doi: 10.1590/S010351502012000400019

KIEVES, N. R.; BERGH, M. S.; ZELLNER, E.; WANG, C. Pilot study measuring the effects of bandaging and cold compression therapy following tibial plateau levelling osteotomy. The Journal of Small Animal Practice, v. 57, n. 10, p. 543-547, 2016. Disponível em: <http://doi.org/10.1111/jsap.12533>. doi: 10.1111/jsap.12533

KIM, J. H.; PARK, H. M. Unilateral femoral arterial thrombosis in a dog with malignant mammary gland tumor: clinical and thermographic findings, and successful treatment with local intra-arterial administration of streptokinase. The Journal of Veterinary Medical Science, v. 74, n. 5, p. 657-661, 2012. Disponível em: <http://doi.org/10.1292/jvms.11-0432>. doi: 10.1292/jvms.11-0432

KONDO, Y.; NAKANO, J.; SAKAMOTO, J.; KATAOKA, H.; YOKOYAMA, S., et al. Effects of Prolonged Stretching and Thermotherapy on Muscle Contracture of Immobilized Rat Soleus Muscle. Journal of Physical Therapy Science, v. 24, n. 6, p. 541-547, 2012. Disponível em: <http://doi.org/10.1589/jpts.24.541>. doi: 10.1589/jpts.24.541

LAHIRI, B. B.; BAGAVATHIAPPAN, S.; JAYAKUMAR, T.; JOHN PHILIP. Medical application of infrared thermography: A review. Infrared Physics \& Technology, v. 55, n. $\quad 4$ p. 221-235, 2012. Disponível em: <https://doi.org/10.1016/j.infrared.2012.03.007>. doi: 10.1016/j.infrared.2012.03.007

LEVINE, D.; MILLIS, D. L.; MYNATT, T. Effects of 3.3-MHz ultrasound on caudal thigh muscle temperature in dogs. Veterinary Surgery, v. 30, n. 2, p. 170-174, 2001. Disponível em: <http://doi.org/10.1053/jvet.2001.21390>. doi: 10.1053/jvet.2001.21390

LEVINE, D.; WATSON, T. Therapeutic Ultrasound. In: Millis, D., Levine, D., editors. Canine rehabilitation and physical therapy. 2 nd edition. Philadelphia: Saunders, $p$. 328-341, 2014.

LIAPI, E.; GESCHWIND, J.F. Hyperthermia - description of a method and a review of clinical applications. Reports of Practical Oncology and Radiotherapy, v. 12, n. 5, p. 267-275, 2007. Disponível em: <https://doi.org/10.1016/S1507-1367(10)60065$X>$. doi: $10.1016 / S 1507-1367(10) 60065-X$

MACHADO, C. M. Eletrotermoterapia prática. $3^{a}$ ed. São Paulo: Pancast Editora; p. 148, 2002. 
MAGGI, L. E.; OMENA, T. P.; VON KRÜGER, M. A.; PEREIRA, W. C. A. Software didático para modelagem do padrão de aquecimento dos tecidos irradiados por ultrasom fisioterapêutico. Revista Brasileira de Fisioterapia, v. 12, n. 3, p. 204-214, 2008. Disponível em: <http://dx.doi.org/10.1590/S1413-35552008000300008>. doi: 10.1590/S1413-35552008000300008

MIKAIL, S.; PEDRO, C. R. Fisioterapia Veterinária. 2. ed. São Paulo: Manole, 2009.

MILLARD, R. P.; TOWLE-MILLARD, H. A; RANKIN, D. C.; ROUSH. J. K. Effect of warm compress aplication on tissue temperature in healthy dogs. American Journal of Veterinary Research, v. 74, n. 3, p. 448-451, 2013a. Disponível em: <http://doi.org/10.2460/ajvr.74.3.448>. doi: 10.2460/ajvr.74.3.448

MILLARD, R. P.; TOWLE-MILLARD, H. A; RANKIN, D. C.; ROUSH. J. K. Effect of cold compress aplication on tissue temperature in healthy dogs. American Journal of Veterinary Research, v. 74, n. 3, p. 443-447, 2013b. Disponível em: <http://doi.org/10.2460/ajvr.74.3.443>. doi: 10.2460/ajvr.74.3.443

MILLIS, D. L.; CIUPERCA, I. A. Evidence for canine rehabilitation and physical therapy. Veterinary Clinics of North America: Small Animal Practice, v. 45, n. 1, p. 1-27, 2015. Disponível em: <http://doi.org/10.1016/j.cvsm.2014.09.001>. doi: 10.1016/j.cvsm.2014.09.001

MONTGOMERY, L; ELLIOTT, S. B.; ADAIR, H. S. Muscle and tendon heating rates with therapeutic ultrasound in horses. Veterinary Surgery, v. 42, n. 3, p. $243-249$, 2013. Disponível em: <http://doi.org/10.1111/j.1532-950X.2013.01099.x>. doi: 10.1111/j.1532-950X.2013.01099.x

MULLER, M. C.; GRADNER, C.; HITTMAIR, K. M.; DUPRE, G.; BOCKSTAHLER, B. A. Conservative treatment of partial gastrocnemius muscle avulsions in dogs using therapeutic ultrasound - A force plate study. Veterinary and Comparative Orthopaedics and Traumatology, v. 22, n. 3, p. 243-248, 2009. Disponível em: $<$ http://doi.org/10.3415/VCOT-08-07-0059>. doi: 10.3415/VCOT-08-07-0059

PANDELEA, AE.; BUDESCU, M. Using thermography and artificial neural networks in civil engineering. Bulletin of the Polytechnic Institute of Jassy, v. 66, n. 2, p. 29-36, 2016. Disponível em: <http://www.bipcons.ce.tuiasi.ro/Archive/557.pdf>.

PAVELSKI, M.; SILVA, D. M.; LEITE, N. C.; JUNIOR, D. A.; DE SOUSA, R. S., et al. Infrared thermography in dogs with mammary tumors and healthy dogs. Journal of Veterinary Internal Medicine, v. 29, n. 6, p. 1570-1583, 2015. Disponível em: <http://doi.org/10.1111/jvim.13597>. doi: 10.1111/jvim.13597

RAMOS, G. V.; PINHEIRO, C. M.; MESSA, S. P.; DELFINO, G. B.; MARQUETI, R. C., et al. Cryotherapy reduces inflammatory response without altering muscle regeneration process and extracelular matrix remodeling of rat muscle. Scientific Reports, v. 6, n. 18525, 2016. Disponível em: <http://dx.doi.org/10.1038/srep18525>. doi: 10.1038/srep18525 
REDAELLI, V.; LUDWIG, N.; COSTA, L. N.; CROSTA, L.; RIVA, J., et al. Potential application of thermography (IRT) in animal production and for animal welfare. A case report of working dogs. Annali dell'Istituto Superiore di Sanità, Roma, v. 50, n. 2, p. 147-152, 2014. Disponível em: <http://doi.org/10.4415/ANN_14_02_07>. doi: 10.4415/ANN_14_02_07

ROBERTO, J. V. B.; SOUZA, B. B. Utilização da termografia de infravermelho na medicina veterinária e na produção animal. Journal of Animal Behaviour and Biometerology, v. 2, n. 3, p. 73-84, 2014. Disponível em: <http://dx.doi.org/10.14269/2318-1265/jabb.v2n3p73-84>. doi: 10.14269/23181265/jabb.v2n3p73-84

SAMOY, Y.; VAN RYSSEN, B.; SAUNDERS, J. Physiotherapy in small animal medicine. Vlaams Diergeneeskundig Tijdschrift, v. 85, n. 6, p. 323-334, 2016. Disponível em: <http://vdt.ugent.be/sites/default/files/01_2.pdf>.

SIMS, C.; WALDRON, R.; MARCELLIN-LITTLE, D. J. Rehabilitation and physical therapy for the neurologic veterinary patient. Veterinary Clinics of North America: Small Animal Practice, v. 45, n. 1, p. 123-143, Jan 2015. Disponível em: <http://doi.org/10.1016/j.cvsm.2014.09.007>. doi: 10.1016/j.cvsm.2014.09.007

STEISS, J. E.; LEVINE, D. Modalidades de Agentes Físicos. In: Levine, D.; Millis, D. L.; Marcellin-Little, D. J.; Taylor, R., editors. Reabilitação e fisioterapia na prática de pequenos animais. São Paulo: Roca, p. 75-94, 2008.

TRAVAIN, T.; COLOMBO, E. S.; GRANDI, L. C.; HEINZL, E.; PELOSI, A.; PRATO PREVIDE, E. P., et al. How good is this food? A study on dogs' emotional responses to a potentially pleasant event using infrared thermography. Physiology \& Behavior, v. 159, p. 80-87, 2016.201 Disponível $<$ https://doi.org/10.1016/j.physbeh.2016.03.019>. doi: 10.1016/j.physbeh.2016.03.019

TRAVAIN, T.; COLOMBO, E. S.; HEINZL, E.; BELLUCCI, D.; PREVIDE, E. P., et al. Hot dogs: Thermography in the assessment of stress in dogs (Canis familiaris)-A pilot study. Journal of Veterinary Behavior: Clinical Applications and Research, v. $10, \quad$ n. $1, \quad$ p. 17-23, 2015. Disponível em: <https://doi.org/10.1016/j.jveb.2014.11.003>. doi: 10.1016/j.jveb.2014.11.003

USUBA, M.; MIYANAGA, Y.; MIYAKAWA, S.; MAESHIMA, T.; SHIRASAKI, Y. Effect of heat in increasing the range of knee motion after the development of a joint contracture: an experiment with an animal model. Archives of Physical Medicine and Rehabilitation, v. 87, n. 2, p. 247-253, 2006. Disponível em: <http://doi.org/10.1016/j.apmr.2005.10.015>. doi: 10.1016/j.apmr.2005.10.015 